\title{
Numerical simulation on aerosol pool scrubbing
}

\author{
Cheng Peng ${ }^{1, *}$, Dong Li $^{1}$, Chengyao Wang ${ }^{l}$, Qinggang Lei ${ }^{1}$ \\ ${ }^{1}$ College of Energy and Mechanical Engineering, Shanghai University of Electric Power, Shanghai, China
}

\begin{abstract}
In the event of severe accident for nuclear power plant (NPP), radioactive aerosols may be released into spent fuel pool (SFP) through filtered-containment-venting-system (FCVS), which are entrained by mixing gas from containment, in order to prevent the overpressure of containment. The efficiency of aerosol pool scrubbing determines the radioactive threats of spent fuel compartment induced by containment venting, and the probable amount of radioactive substance during atmospheric dispersion later on. Therefore, it is necessary to study the typical flow phenomena during the process of aerosol pool scrubbing in the SFP of PWR nuclear power plants, and figure out the important regularities and mechanisms, which can provide reference for evaluations of radioactive threats of spent fuel compartment and provide technical supports for new type of designs for SFP and venting system. In this paper, Fluent is used to establish two kinds of numerical models of SFP, including horizontal injection and vertically downward injection configurations, according to the geometrical dimensions of Qinshan 2 NPP's SFP. $\mathrm{TiO}_{2}$ is used as the substitute of radioactive substance and coupled numerical models of VOF and DPM are introduced for qualitative and quantitative studies on the effects of diameter of aerosol particle, injection velocity, initial water depth and injection direction. The decontamination factor (DF) is determined by quantifying the mass concentration of aerosol particles which escape and are traced from the SFP under different conditions, respectively. Based on the simulation results, it can be seen that with the increase of particle diameter, DF is going to decrease at first and then increase. There should be a negative correlation between injection velocity and DF. By contrast, there is a positive association between initial water depth and DF. Besides, DFs under vertically downward injection conditions are much lower than that under horizontal injection, due to the appearance of contra-vortex flow adjacent to the free water surface.
\end{abstract}

\section{Introduction}

Core melt-down in severe accident may release large number of radioactive aerosols [1], which disperse in the atmosphere of containment (filled with air) along with steam, when the integrity of reactor coolant system (RCS) is damaged. This kind of ingress may result in overpressure of the containment [2]. In order to protect the last barrier of NPP, the filtered-containment-ventingsystem (FCVS) is designed to effectively mitigate this potential risk and eliminate hazardous radioactive release as much as possible, the operating principle of which is that the mixing gas within the containment is drained through automatic depressurization, meanwhile, the radioactive substance involved is removed when it passes through a filter installed in the system, in case of decontaminating the environment [3]. Spent fuel pool (SFP), as shown in Fig.1, which is treated as the terminal station of FCVS, has drawn experts' attention, since Fukushima nuclear accident, including the study on long-term passive cooling characters of SFP [4]. However, in consideration of lowering the risk of severe accident happened in SFP, it is necessary to investigate the aerosol pool scrubbing behaviors during depressurization, which can also provide reference for evaluations of radioactive threats of spent fuel compartment and provide technical supports for new type of designs of SFP and venting system.

After the radioactive aerosols enter into the SFP, along with large quantity of non-condensable gas (air) and little condensable gas (steam), a part of them may be deposited by the coupled effects of injection momentum, gravity, turbulence [5] and surface adhesion [6]. During this process, diameter of aerosol particles, injection velocity, initial water depth and injection direction can be the significant factors that dominate the scrubbing effect. Therefore, it's very important to get better undstandings of the effects of dominant factors on aerosol pool scrubbing, so as to optimze the design of SFP.

\footnotetext{
* Corresponding author: diomio@shiep.edu.cn
} 


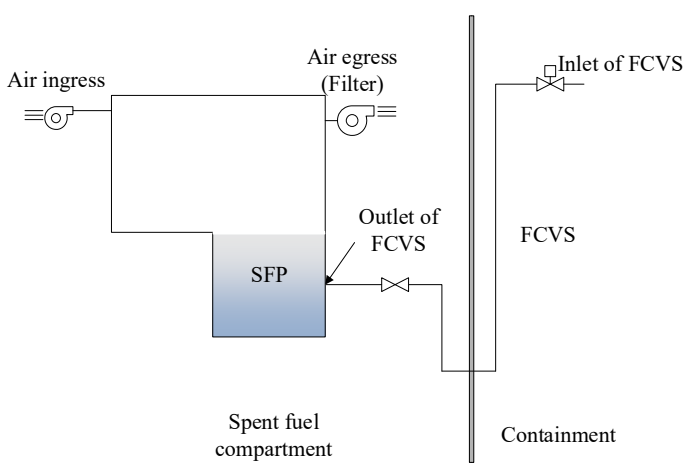

Fig. 1. The relation between FCVS in containment and SFP in spent fuel compartment

Since last century, studies on natural deposition and scrubbing effect of fission product aerosol have been carried out. Huang [7] evaluated the contribution of gravitational sedimentation, inertial impaction, diffusionphoresis, and thermophoresis to aerosol deposition in typical severe accidents, based on integrated safety analysis model. Moreover, Chen [8] used CFD method to focus on the effects of inejction velocity and diameter of aerosol particles on gravitational sedimentation in gaseous phase. Besides, Siemens [9] designed a Venturi-tube-scrubbing-system, and purified the SFP by fluid disturbance generated from a spray nozzle. Yang [10] calculated particle deposition rate in liquid by adopting Fluent code, and concluded that there should be a positive relation between the deposition rate of tiny particles and mean velocity. Zhao [11] conducted numerical research on hydraulic characteristics of solid-liquid two-phase flow in inclined plate sedimentation tank, and established reliable turbulence model (RNG $k-\varepsilon$ ) and multi-phase model (Mixture) by validating agasint experimental data. Then, the relation between particle diameter and decontamination rate in sedimentation pool was determined. However, these investigations referred above just focus on effect of single variable in conventional industries or natural deposition regimes. For SFP in nuclear industry, the inejction velocity of aerosol particles depend on depressurization in severe accident, and the diameter of aerosol particles ranges from $10^{0}$ to $10^{1} \mu \mathrm{m}$. Besides, the initial water level is dependent upon thermal-hydraulic conditions of SFP. How does these complex conditions determine the pool scrubbing still remains unknown. Therefore, in this paper, coupled models of VOF and DPM in Fluent code are implemented to study the main influencing factors and the simulation results are verified by literature findings. Based on which, mechanisms involved are analyzed.

Table 1. Geometrical dimensions of Qinshan 2 NPP's SFP.

\begin{tabular}{ccc}
\hline Height $/ \mathbf{m}$ & Diameter/m & $\begin{array}{c}\text { Initial water } \\
\text { depth } / \mathbf{m}\end{array}$ \\
\hline 12.010 & 11.329 & 11.500 \\
\hline
\end{tabular}

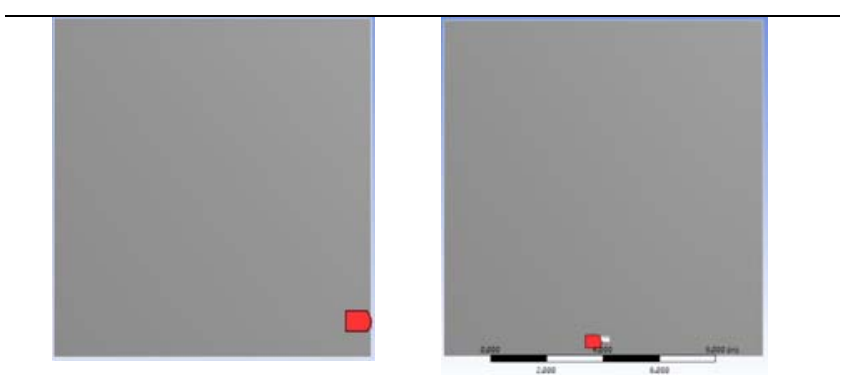

Fig.2. SFP under horizontal injection

Fig.3. SFP under vertically downward injection

\section{SFP scrubbing model and conditions}

\subsection{Geometry modelling}

The geometrical dimensions of Qinshan 2 NPP's SFP are listed in Table 1, and based on which, two kinds of numerical models of SFP are established, including twodimensional horizontal injection (as shown in Fig. 2) and vertically downward injection (as shown in Fig. 3) configurations.

\subsection{Parameters selection and decontamination factor}

In the event of severe accident, aerosol concentration may decrease with time, due to the natural deposition within the containment. When automatic depressurization is introduced, aerosol concentration can be within the range of $0.0478 \sim 0.88 \mathrm{~g} / \mathrm{m}^{3}$ [13]. Besides, with the help of the advice obtained from aerosol removal tests, $\mathrm{TiO}_{2}[13]$ can be used as the substitute of radioactive substance, with the density of $3850 \mathrm{~kg} / \mathrm{m}^{3}$. For the diameter of aerosol particles, the median diameter of $1 \mu \mathrm{m}$ is selected as the base, which is referred from Siemens's investigation on aerosol size distribution in severe accident.

After mixing gas carries the aerosols into SFP, noncondensable gas gradully forms bubbles to move upwards, meanwhile, aerosols can be deposited in the pool by various mechanisms. In order to estimate the scrubbing effect of SFP under different conditions, mass concentrations of aerosol at inlet and outlet of SFP are traced, thus determining the decontamination factor, DF, which can be described as equation (1).

$$
D F=\frac{m_{1}}{m_{2}}
$$

Where $m_{1}$ and $m_{2}$ refer to the mass concentrations of aerosol at inlet and outlet of SFP, respectively.

Table 2. Grid parameters.

\begin{tabular}{ccc}
\hline Case. & $\begin{array}{c}\text { Grid } \\
\text { numbers }\end{array}$ & Standard deviation \\
\hline Grid-1 & 2475 & $1.62 \mathrm{e}-002$ \\
Grid-2 & 25756 & $1.55 \mathrm{e}-005$ \\
Grid-3 & 262856 & $9.61 \mathrm{e}-006$ \\
\hline
\end{tabular}


Table 3. DFs for different grids.

\begin{tabular}{cccc}
\hline Case. & $\begin{array}{c}\text { Aerosol } \\
\text { numbers at } \\
\text { inlet }\end{array}$ & $\begin{array}{c}\text { Aerosol } \\
\text { numbers at } \\
\text { outlet }\end{array}$ & DF \\
\hline Grid-1 & 50 & 26 & 1.92 \\
Grid-2 & 250 & 62 & 4.03 \\
Grid-3 & 300 & 74 & 4.05 \\
\hline
\end{tabular}

\subsection{Grid sensitivity analysis}

For the purpose of ensuring the accuracy of simulations and saving the computer resources as many as possible, different numbers of grid are employed for the horizontal injection configuration, as shown in Table 2. The initial conditions include the injection velocity of $2 \mathrm{~m} / \mathrm{s}$, with the aerosol particle of $10 \mu \mathrm{m}$ and initial water depth of $11.5 \mathrm{~m}$. The comparison is shown in Table 3, where the DF in Case. grid-1 is far less than that in both Case. grid2 and Case. grid-3, and the DF in Case. Grid-2 is more similar to that in Case. grid-3. Allowing for the time saving and accuracy of simulations, the grid number of 25756 is chosen for the following research.

\subsection{Simulation conditions}

The simulation conditions are demonstrated in Table 4, in which the base cases for horizontal inejction and vertically downward injection configurations are Case 1 and Case 8, respectively. Others are used for sensitivity analyses, including particle diameter, injection velocity, initial water depth and injection direction. In this paper, we simplify the real pool scrubbing process by neglecting the heat transfer from the spent fuel stored and thermophoresis, as well. Only deposition mechanisms which associate with hydraulics are concentrated on.

Table 4. Simulation conditions of aerosol pool scrubbing.

\begin{tabular}{|c|c|c|c|c|}
\hline Case. & $\begin{array}{c}\text { Injection } \\
\text { velocity } \\
(\mathrm{m} / \mathbf{s})\end{array}$ & $\begin{array}{c}\text { Particle } \\
\text { diameter } \\
(\mu \mathrm{m})\end{array}$ & $\begin{array}{c}\text { Initial } \\
\text { water } \\
\text { depth } \\
\text { (m) }\end{array}$ & $\begin{array}{l}\text { Injection } \\
\text { direction }\end{array}$ \\
\hline 1 & 2 & 1 & 11.5 & Horizontal \\
\hline 2 & 2 & 5 & 11.5 & Horizontal \\
\hline 3 & 2 & 10 & 11.5 & Horizontal \\
\hline 4 & 4 & 1 & 11.5 & Horizontal \\
\hline 5 & 8 & 1 & 11.5 & Horizontal \\
\hline 6 & 2 & 1 & 6.5 & Horizontal \\
\hline 7 & 2 & 1 & 1.5 & Horizontal \\
\hline 8 & 2 & 1 & 11.5 & $\begin{array}{l}\text { Vertically } \\
\text { downward }\end{array}$ \\
\hline 9 & 2 & 5 & 11.5 & $\begin{array}{l}\text { Vertically } \\
\text { downward }\end{array}$ \\
\hline 10 & 2 & 10 & 11.5 & $\begin{array}{l}\text { Vertically } \\
\text { downward }\end{array}$ \\
\hline 11 & 4 & 1 & 11.5 & $\begin{array}{l}\text { Vertically } \\
\text { downward }\end{array}$ \\
\hline 12 & 8 & 1 & 11.5 & $\begin{array}{l}\text { Vertically } \\
\text { downward }\end{array}$ \\
\hline 13 & 2 & 1 & 6.5 & $\begin{array}{l}\text { Vertically } \\
\text { downward }\end{array}$ \\
\hline 14 & 2 & 1 & 1.5 & $\begin{array}{l}\text { Vertically } \\
\text { downward }\end{array}$ \\
\hline
\end{tabular}

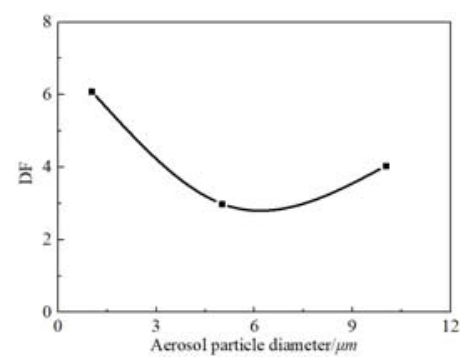

Fig.4. Effect of aerosol particle diameter on pool scrubbing

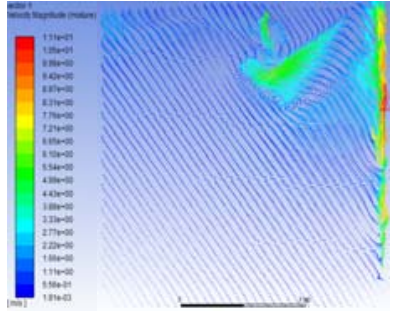

Fig.5. Velocity distribution under aerosol particle diameter of $1 \mu \mathrm{m} \quad(t=5 \mathrm{~s})$

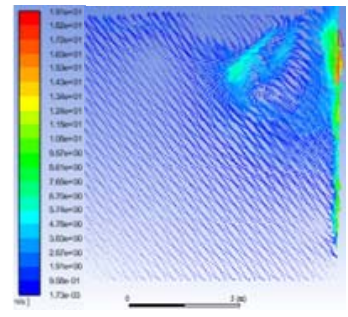

Fig.6. Velocity distribution under aerosol particle diameter of $5 \mu \mathrm{m} \quad(t=5 \mathrm{~s})$

\section{Analysis on main influencing factors}

\subsection{Effect of aerosol particle diameter on pool scrubbing}

In Table 4, the aerosol particle diameters are 1, 5 and 10 $\mu \mathrm{m}$ in Case 1, 2 and 3, respectively. The initial water depth and injection velocity are kept constant, and simulation reuslts are shown in Fig. 4-6.

It can be seen that DF is going to decrease at first and then increase. For the tiny particles $(d<5 \mu \mathrm{m})$, as shown in Fig.5 and Fig.6, the velocity magnitude under particle diameter of $1 \mu \mathrm{m}$ is less than that under $5 \mu \mathrm{m}$, thus leading to the longer duration of aerosols' transport within the water pool. Therefore, DF is much larger under particle diameter of $1 \mu \mathrm{m}$ for the same time. However, when the particle diameter increases to a certain extent $(d>5 \mu \mathrm{m})$, inertial impaction may dominate [16] during pool scrubbing, and aerosols can be blocked and captured by liquidus phase easily for the same injection velocity, which has been verified by previous experimental study [17].

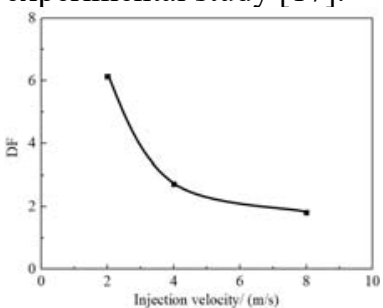

Fig.7. Effect of injection velocity on pool scrubbing

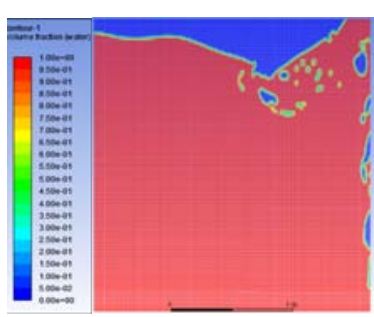

Fig.8. Volume fraction of water under injection velocity of $2 \mathrm{~m} / \mathrm{s} \quad(t=5 \mathrm{~s})$ 


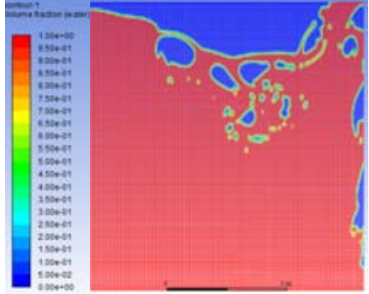

Fig.9. Volume fraction of water under injection velocity of $4 \mathrm{~m} / \mathrm{s} \quad(t=5 \mathrm{~s})$

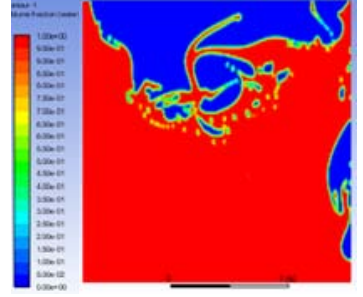

Fig.10. Volume fraction of water under injection velocity of $10 \mathrm{~m} / \mathrm{s} \quad(t=5 \mathrm{~s})$

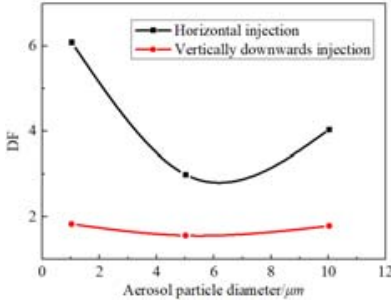

Fig.15. Effect of aerosol particle diameter

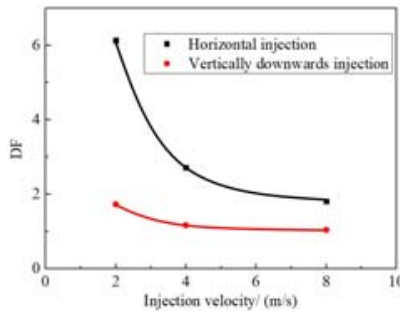

Fig.17. Effect of injection velocity

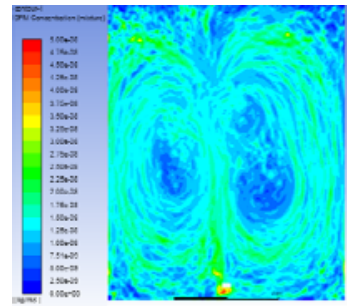

Fig.16. Mass concentration distribution of aerosols under particle diameter of $1 \mu \mathrm{m}$ $(t=5 \mathrm{~s})$

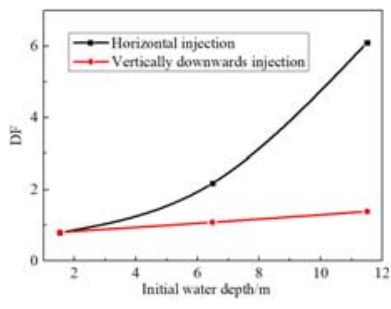

Fig.18. Effect of initial water depth of phenomenon can be explained by the coupled effects of inertial impaction and turbulent flow induced by the rise of non-condensable bubbles. As discussed in section 3.1, the larger the injection velocity, the more the fraction of deposition caused by inertial impaction. However, the residence time for bubbles decreases. Besides, what can be found from Fig. 8-10 is that the free surface of water is unstable and may deform when bubbles move upwards, thus leading to the decrease of water level in some local regions, which helps aerosols escape from SFP. Therefore, the limited residence time and deformation of the free water surface contribute to the decrease of DF.

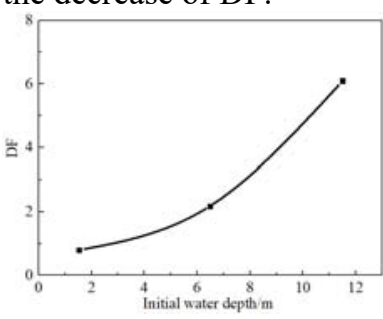

Fig.11. Effect of initial water depth on pool scrubbing

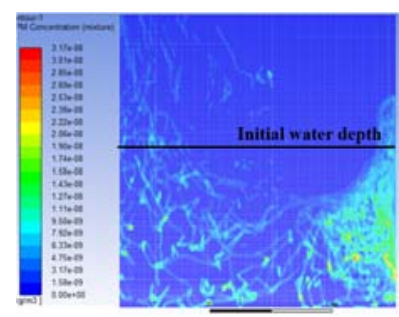

Fig.13. Mass concentration distribution of aerosols under initial water depth of $6.5 \mathrm{~m}$ $(t=5 \mathrm{~s})$

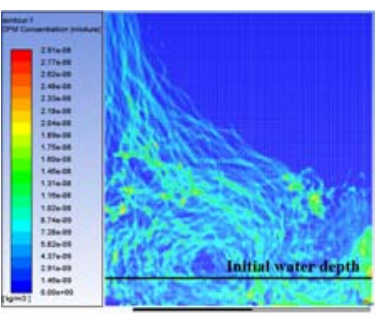

Fig.12. Mass concentration distribution of aerosol under initial water depth of $1.5 \mathrm{~m}$ $(t=5 \mathrm{~s})$

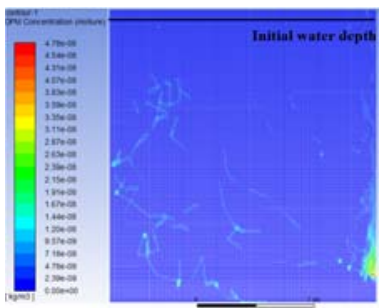

Fig.14. Mass concentration distribution of aerosols under initial water depth of $11.5 \mathrm{~m}$ $(t=5 \mathrm{~s})$

\subsection{Effect of initial water depth on pool scrubbing}

In Table 4, the initial water depths are 11.5, 6.5 and $1.5 \mathrm{~m}$ in Case 1, 6 and 7, respectively. The injection velocity and aerosol particle diameter are kept constant and simulation reuslts are shown in Fig. 11-14.

It can be concluded that DF increases with the increase of initial water depth, which is in line with the POSEIDON-II test results and simualtion results obtained from BUSCA code [19]. The mass concentration distribution of aerosol at $t=5 \mathrm{~s}$ can be shown in Fig. 12-14, which presents that the mass fraction of aerosol at the exit of SFP can decrease with the increase of initial water depth for the same period of time, due to the adhesive force of water. Furthermore, the injection momentum may deform the free surface of water, when the initial water depth is much lower, thus strengthening aerosols' escape (as shown in Fig. 12), as analysed in section 3.2 .

\subsection{Comparison between vertically downward injection and horizontal injection}

In this article, vertically downward injection conditions are still discussed, compared with those under horizontal injections, in order to provide useful information for potential optimization against arrangments of filtered venting pipes in NPPs. Simulation results have been shown in Fig. 15-22. 


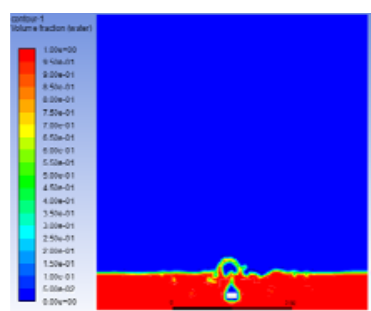

Fig.19. Volume fraction of water under initial water depth of $1.5 \mathrm{~m} \quad(t=5 \mathrm{~s})$

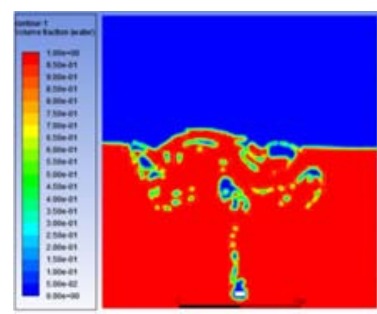

Fig.21. Volume fraction of water under initial water depth of $6.5 \mathrm{~m} \quad(t=5 \mathrm{~s})$

It can be seen from Fig. 15 that the effect of aerosol partcle diameter on pool scubbing under vertically downward injection is similar to that under horizontal injection, however, the DF magnitute is much less, which attributes to the two vortexes formed symmetrically in SFP, as shown in Fig. 16. Even though they can enhance the mixing of aerosols and the fluid, they can still carry large numbers of aerosol particles in the bottom of the pool to the free surface of water, as well. Therefore, the entrainments from two vortexes in SFP may reduce the DF, which should be paid much attention in SFPs' design.

For the effect of injection velocity and initial water depth, similar results can also be acquired, as shown in Fig. 17-18, with much lower values than those under corresponding conditions. Volume fraction of water and velocity distribution under initial water depth of $1.5 \mathrm{~m}$ and $6.5 \mathrm{~m}$ at $t=5 \mathrm{~s}$ are given in Fig. 19-22. It can be found that the free surface of water is much flatter than that under horizontal injections, due to the less effect of inertial impaction, after the jet is blocked and the corresponding injection momentum decreases rapidly. Besides, the two vortexes exist not only beneath the free surface of water, but also in the gaseous phase, which can be even more beneficial to the particles' escape from the pool.

\section{Conclusions}

Aerosol pool scrubbing is a very important process during filtered venting in nuclear power plants. In this paper, coupled numerical models of VOF and DPM are introduced to investigate the hydraulic phenomena under both of horizontal and vertically downwards injection conditions. By varying aerosol particle diameter, injection velocity and initial water depth of SFP, the effects of main influencing factors on DF and mechanisms involved are studied. The main conclusions are drawn as below.

1) With the increase of aerosol particle diameter, DF is going to decrease at first and then increase.

2) There should be a negative correlation between injection velocity and DF. By contrast, there is a positive association between initial water depth and DF.

3) Besides, DFs under vertically downward injection conditions are much lower than that under horizontal injections, due to the appearance of contra-vortex flow adjacent to the free water surface, which strengthens the escape of aerosols.

\section{References}

1. K. Knebel, D. Bottomley, V.V. Rondinella, A. Lähde, J. Jokiniemi, J. Aerosol. Sci. 106 (2017)

2. R.H. Chen, P.H. Zhang, P. Ma, B. Tan, Z.L. Wang, D. Zhang, W.X. Tian, S.Z. Qiu, G.H. Su, Ann. Nucl. Energy. 136 (2020)

3. L.L. Tong, Nuclear reactor severe accident mechanism research. (2016)

4. M.L. Wang, Experimental study on the heat pipe for the long-term passive cooling system of spent fuel pool. (2014)

5. B.J. Merrill, D.L. Hagrman, MELCOR aerosol transport module modification for NSSR-1. (INEL96/0081, 1996)

6. H. Allelein, A. Auvinen, J. Ball, S. Güntay, L.E. Herranz, A. Hidaka, A.V. Jones, M. Kissane, D. Powers, G. Weber, State of the art report on nuclear aerosols. (NEA/CSNI/R(2009)5, 2009)

7. G.F. Huang, X.W. Cao, L.L. Tong, Nucl. Power. Eng. 33, 04 (2012)

8. L.L. Chen, Sci. Technol. Info. 12, 20 (2014)

9. Rust, Ch. Tännler, W. Heintz, D. Haschke, M. Nuala, R. Jakab, Nucl. Eng. Des. 157, (1995)

10. X. Yang, The difference of fine particle deposition in the gas and liquid medium. (2016)

11. D.X. Zhao, Numerical simualtion of hydraulic characteristics of solid-liquid two-phase flow in inclined plate sedimentation tank. (2019)

12. X.L. Shi, Y.C. Zhang, Y.S. Wei, Technol. Innov. App. 23, (2019)

13. X.F. Zhou, H.F. Gu, F. L, Nucl. Power. Eng. 5, (2014)

14. Losch, V. Tiegs, B. Eckardt, Qinshan 2 NPP's containment venting system. (Qinshan: Framatome ANP GmbH, 2003)

15. M. Berlin, M. Delalande, Research and development on the venting and filtering system for pressurized water reactor containments. (Specialist Meeting on Filtered Containment Venting Systems, CSNI Report 148. Paris: OCED Nuclear Energy Agency, 1988) 
16. P.C. Owczarski, K.W. Burk, SPARC-90: A code for calculating fission product capture in suppression pools. (Nuclear Regulatory Commission, Washington, DC (United States). 1991)

17. Y.M. Zhou, H.F. Gu, Q.N. Sun, Z.N. Sun, J.Q. Su, L. Gao, Q.C. Ma, G. Zhu, Y.Z. Li, Research on deposition of micro-nano aerosols in rising bubble under pool scrubbing condition. (2018 26th International Conference on Nuclear Engineering: American Society of Mechanical Engineers, 2018)

18. M. Turni, Experimental study and modeling of a pool scrubbing system for aerosol removal. (2016)

19. Dehbi, D. Suckow, S. Guentay, Nucl. Eng. Des. 203, 2-3 (2001) 